\title{
A New Extension of Hardy-Hilbert's Inequality in the Whole Plane
}

\author{
Bicheng Yang ${ }^{1}$ and Qiang Chen ${ }^{2}$ \\ ${ }^{1}$ Department of Mathematics, Guangdong University of Education, Guangzhou, Guangdong 510303, China \\ ${ }^{2}$ Department of Computer Science, Guangdong University of Education, Guangzhou, Guangdong 510303, China
}

Correspondence should be addressed to Bicheng Yang; bcyang818@163.com

Received 27 March 2016; Accepted 30 June 2016

Academic Editor: Raúl E. Curto

Copyright (C) 2016 B. Yang and Q. Chen. This is an open access article distributed under the Creative Commons Attribution License, which permits unrestricted use, distribution, and reproduction in any medium, provided the original work is properly cited.

By the use of weight coefficients and Hermite-Hadamard's inequality, a new extension of Hardy-Hilbert's inequality in the whole plane with multiparameters and a best possible constant factor is given. The equivalent forms, the operator expressions, and a few particular inequalities are considered.

\section{Introduction}

Suppose that $p>1,1 / p+1 / q=1, a_{m}, b_{n} \geq 0, a=\left\{a_{m}\right\}_{m=1}^{\infty} \in$ $l^{p}, b=\left\{b_{n}\right\}_{n=1}^{\infty} \in l^{q},\|a\|_{p}=\left(\sum_{m=1}^{\infty} a_{m}^{p}\right)^{1 / p}>0$, and $\|b\|_{q}>0$. We have the following well known Hardy-Hilbert's inequality:

$$
\sum_{n=1}^{\infty} \sum_{m=1}^{\infty} \frac{a_{m} b_{n}}{m+n}<\frac{\pi}{\sin (\pi / p)}\|a\|_{p}\|b\|_{q},
$$

where the constant factor $\pi / \sin (\pi / p)$ is the best possible one (cf. [1]). The more accurate form of (1) was given as follows (cf. [2], Theorem 323):

$$
\begin{aligned}
\sum_{n=1}^{\infty} \sum_{m=1}^{\infty} \frac{a_{m} b_{n}}{m+n-2 \alpha}<\frac{\pi}{\sin (\pi / p)}\|a\|_{p}\|b\|_{q} & \\
& \left(0 \leq \alpha \leq \frac{1}{2}\right),
\end{aligned}
$$

where the constant factor $\pi / \sin (\pi / p)$ is still the best possible one. For $\alpha=0$, inequality (2) reduces to (1).
In 2011, Yang gave an extension of (2) as follows (cf. [3]): If $0<\lambda_{1}, \lambda_{2} \leq 1, \lambda_{1}+\lambda_{2}=\lambda, a_{m}, b_{n} \geq 0,\|a\|_{p, \varphi}=$ $\left\{\sum_{m=1}^{\infty}(m-\alpha)^{p\left(1-\lambda_{1}\right)-1} a_{m}^{p}\right\}^{1 / p} \in(0, \infty)$, and $\|b\|_{q, \psi}=\left\{\sum_{n=1}^{\infty}(n-\right.$ $\left.\alpha)^{q\left(1-\lambda_{2}\right)-1} b_{n}^{q}\right\}^{1 / q} \in(0, \infty)$, then

$$
\begin{aligned}
\sum_{n=1}^{\infty} \sum_{m=1}^{\infty} \frac{a_{m} b_{n}}{(m+n-2 \alpha)^{\lambda}}<B\left(\lambda_{1}, \lambda_{2}\right)\|a\|_{p, \varphi} & \|b\|_{q, \psi} \\
& \left(0 \leq \alpha \leq \frac{1}{2}\right),
\end{aligned}
$$

where the constant factor $B\left(\lambda_{1}, \lambda_{2}\right)$ is the best possible one and $B(u, v)$ is the beta function defined by (cf. [4])

$$
B(u, v):=\int_{0}^{\infty} \frac{1}{(1+t)^{u+v}} t^{u-1} d t \quad(u, v>0)
$$

For $\lambda=1, \lambda_{1}=1 / q$, and $\lambda_{2}=1 / p$, (3) reduces to (2). Some other results related to (1)-(3) are provided by [5-22].

In this paper, by the use of weight coefficients and Hermite-Hadamard's inequality, an extension of (3) in the 
whole plane is given as follows: For $\xi, \eta \in[0,1 / 2], a_{m}, b_{n} \geq$ $0, \sum_{|m|=1}^{\infty}|m-\xi|^{p\left(1-\lambda_{1}\right)-1} a_{m}^{p} \in(0, \infty)$, and $\sum_{|n|=1}^{\infty} \mid n-$ $\left.\eta\right|^{q\left(1-\lambda_{2}\right)-1} b_{n}^{q} \in(0, \infty)$, we have

$$
\begin{gathered}
\sum_{|n|=1}^{\infty} \sum_{|m|=1}^{\infty} \frac{1}{(|m-\xi|+|n-\eta|)^{\lambda}} a_{m} b_{n}<2 B\left(\lambda_{1}, \lambda_{2}\right) \\
\cdot\left[\sum_{|m|=1}^{\infty}|m-\xi|^{p\left(1-\lambda_{1}\right)-1} a_{m}^{p}\right]^{1 / p} \\
\cdot\left[\sum_{|n|=1}^{\infty}|n-\eta|^{q\left(1-\lambda_{2}\right)-1} b_{n}^{q}\right]^{1 / q} .
\end{gathered}
$$

Moreover, a generation of (5) with multiparameters and a best possible constant factor is proved. The equivalent forms, the operator expressions, and a few particular inequalities are also considered.

\section{Some Lemmas}

First, we make appointment that $\mathbf{N}=\{1,2, \ldots\}, p>1,1 / p+$ $1 / q=1, \alpha, \beta \in(0, \pi), \xi, \eta \in[0,1 / 2], \lambda_{1}, \lambda_{2}>0, \lambda_{1}+\lambda_{2}=\lambda$, and, for $|x|,|y|>1 / 2$,

$$
\begin{aligned}
& k(x, y) \\
& :=\frac{1}{[|x-\xi|+(x-\xi) \cos \alpha+|y-\eta|+(y-\eta) \cos \beta]^{\lambda}} .
\end{aligned}
$$

In particular, for $\alpha=\beta=\pi / 2$, we indicate

$$
h(x, y):=\frac{1}{(|x-\xi|+|y-\eta|)^{\lambda}}, \quad|x|,|y|>\frac{1}{2} .
$$

Definition 1. Define the following weight coefficients:

$$
\begin{aligned}
& \omega\left(\lambda_{2}, m\right):=\sum_{|n|=1}^{\infty} k(m, n) \frac{[|m-\xi|+(m-\xi) \cos \alpha]^{\lambda_{1}}}{[|n-\eta|+(n-\eta) \cos \beta]^{1-\lambda_{2}}}, \\
& |m| \in \mathbf{N}, \\
& \omega\left(\lambda_{1}, n\right) \\
& :=\sum_{|m|=1}^{\infty} k(m, n) \frac{[|n-\eta|+(n-\eta) \cos \beta]^{\lambda_{2}}}{[|m-\xi|+(m-\xi) \cos \alpha]^{1-\lambda_{1}}},
\end{aligned}
$$

where $\sum_{|j|=1}^{\infty} \cdots=\sum_{j=-1}^{-\infty} \cdots+\sum_{j=1}^{\infty} \cdots(j=m, n)$.

Lemma 2. If $\lambda_{2} \leq 1$, then, for $k_{\beta}\left(\lambda_{1}\right):=2 B\left(\lambda_{1}, \lambda_{2}\right) \csc ^{2} \beta$, one has

$$
k_{\beta}\left(\lambda_{1}\right)\left(1-\theta\left(\lambda_{2}, m\right)\right)<\omega\left(\lambda_{2}, m\right)<k_{\beta}\left(\lambda_{1}\right),
$$

$|m| \in \mathbf{N}$, where

$$
\begin{aligned}
& \theta\left(\lambda_{2}, m\right):=\frac{1}{B\left(\lambda_{1}, \lambda_{2}\right)} \\
& \quad \cdot \int_{0}^{(1+\eta)(1+\cos \beta) /(|m-\xi|+(m-\xi) \cos \alpha)} \frac{u^{\lambda_{2}-1}}{(1+u)^{\lambda}} d u \\
& \quad=O\left(\frac{1}{[|m-\xi|+(m-\xi) \cos \alpha]^{\lambda_{2}}}\right) \in(0,1),
\end{aligned}
$$

$|m| \in \mathbf{N}$.

Proof. For $|x|>1 / 2$, we set

$$
\begin{array}{r}
k^{(1)}(x, y) \\
:=\frac{1}{[|x-\xi|+(x-\xi) \cos \alpha+(y-\eta)(\cos \beta-1)]^{\lambda}}, \\
k^{(2)}(x, y) \\
:=\frac{1}{[|x-\xi|+(x-\xi) \cos \alpha+(y-\eta)(1+\cos \beta)]^{\lambda}}, \\
y>\frac{1}{2},
\end{array}
$$

and then, for $y>1 / 2$,

$$
\begin{aligned}
k^{(1)} & (x,-y) \\
& =\frac{1}{[|x-\xi|+(x-\xi) \cos \alpha+(y+\eta)(1-\cos \beta)]^{\lambda}} .
\end{aligned}
$$

We find

$\omega\left(\lambda_{2}, m\right)$

$$
\begin{aligned}
= & \sum_{n=-1}^{-\infty} k^{(1)}(m, n) \frac{[|m-\xi|+(m-\xi) \cos \alpha]^{\lambda_{1}}}{[(n-\eta)(\cos \beta-1)]^{1-\lambda_{2}}} \\
& +\sum_{n=1}^{\infty} k^{(2)}(m, n) \frac{[|m-\xi|+(m-\xi) \cos \alpha]^{\lambda_{1}}}{[(n-\eta)(1+\cos \beta)]^{1-\lambda_{2}}}
\end{aligned}
$$

$$
\begin{gathered}
=\frac{[|m-\xi|+(m-\xi) \cos \alpha]^{\lambda_{1}}}{(1-\cos \beta)^{1-\lambda_{2}}} \sum_{n=1}^{\infty} \frac{k^{(1)}(m,-n)}{(n+\eta)^{1-\lambda_{2}}} \\
+\frac{[|m-\xi|+(m-\xi) \cos \alpha]^{\lambda_{1}}}{(1+\cos \beta)^{1-\lambda_{2}}} \sum_{n=1}^{\infty} \frac{k^{(2)}(m, n)}{(n-\eta)^{1-\lambda_{2}}} .
\end{gathered}
$$

It is evident that, for fixed $m \in \mathbf{N}, \lambda_{2} \leq 1(\lambda>0)$, both $k^{(1)}(m,-y) /(y+\eta)^{1-\lambda_{2}}$ and $k^{(2)}(m, y) /(y-\eta)^{1-\lambda_{2}}$ are strictly 
decreasing and strictly convex with respect to $y \in(1 / 2, \infty)$, satisfying

$$
\begin{gathered}
\frac{d}{d y} \frac{k^{(i)}\left(m,(-1)^{i} y\right)}{\left[y+(-1)^{i} \eta\right]^{1-\lambda_{2}}}<0, \\
\frac{d^{2}}{d y^{2}} \frac{k^{(i)}\left(m,(-1)^{i} y\right)}{\left[y+(-1)^{i} \eta\right]^{1-\lambda_{2}}}>0,
\end{gathered}
$$

$$
(i=1,2)
$$

By Hermite-Hadamard's inequality (cf. [23]), we find

$$
\begin{aligned}
& \omega\left(\lambda_{2}, m\right) \\
& <\frac{[|m-\xi|+(m-\xi) \cos \alpha]^{\lambda_{1}}}{(1-\cos \beta)^{1-\lambda_{2}}} \int_{1 / 2}^{\infty} \frac{k^{(1)}(m,-y)}{(y+\eta)^{1-\lambda_{2}}} d y \\
& \quad+\frac{[|m-\xi|+(m-\xi) \cos \alpha]^{\lambda_{1}}}{(1+\cos \beta)^{1-\lambda_{2}}} \int_{1 / 2}^{\infty} \frac{k^{(2)}(m, y)}{(y-\eta)^{1-\lambda_{2}}} d y \\
& \leq \frac{[|m-\xi|+(m-\xi) \cos \alpha]^{\lambda_{1}}}{(1-\cos \beta)^{1-\lambda_{2}}} \int_{-\eta}^{\infty} \frac{k^{(1)}(m,-y)}{(y+\eta)^{1-\lambda_{2}}} d y \\
& +\frac{[|m-\xi|+(m-\xi) \cos \alpha]^{\lambda_{1}}}{(1+\cos \beta)^{1-\lambda_{2}}} \int_{\eta}^{\infty} \frac{k^{(2)}(m, y)}{(y-\eta)^{1-\lambda_{2}}} d y .
\end{aligned}
$$

Setting $u=((y+\eta)(1-\cos \beta) /(|m-\xi|+(m-\xi) \cos \alpha))((y-$ $\eta)(1+\cos \beta) /(|m-\xi|+(m-\xi) \cos \alpha))$ in the above first (second) integral, by simplification, we find

$$
\begin{aligned}
\omega\left(\lambda_{2}, m\right) & <\left(\frac{1}{1-\cos \beta}+\frac{1}{1+\cos \beta}\right) \int_{0}^{\infty} \frac{u^{\lambda_{2}-1}}{(1+u)^{\lambda}} d u \\
& =2 B\left(\lambda_{1}, \lambda_{2}\right) \csc ^{2} \beta=k_{\beta}\left(\lambda_{1}\right) .
\end{aligned}
$$

By (14), since both $k^{(1)}(m,-y) /(y+\eta)^{1-\lambda_{2}}$ and $k^{(2)}(m, y) /$ $(y-\eta)^{1-\lambda_{2}}$ are strictly decreasing, we still have

$$
\begin{aligned}
& \omega\left(\lambda_{2}, m\right)>\frac{[|m-\xi|+(m-\xi) \cos \alpha]^{\lambda_{1}}}{(1-\cos \beta)^{1-\lambda_{2}}} \\
& \quad \cdot \int_{1}^{\infty} \frac{k^{(1)}(m,-y)}{(y+\eta)^{1-\lambda_{2}}} d y \\
& \quad+\frac{[|m-\xi|+(m-\xi) \cos \alpha]^{\lambda_{1}}}{(1+\cos \beta)^{1-\lambda_{2}}} \int_{1}^{\infty} \frac{k^{(2)}(m, y)}{(y-\eta)^{1-\lambda_{2}}} d y \\
& \quad \geq \frac{1}{1-\cos \beta}
\end{aligned}
$$

$$
\begin{aligned}
& \cdot \int_{(1+\eta)(1+\cos \beta) /(|m-\xi|+(m-\xi) \cos \alpha)}^{\infty} \frac{u^{\lambda_{2}-1}}{(1+u)^{\lambda}} d u \\
& +\frac{1}{1+\cos \beta} \\
& \cdot \int_{(1+\eta)(1+\cos \beta) /(|m-\xi|+(m-\xi) \cos \alpha)}^{\infty} \frac{u^{\lambda_{2}-1}}{(1+u)^{\lambda}} d u \\
& =k_{\beta}\left(\lambda_{2}\right)\left(1-\theta\left(\lambda_{2}, m\right)\right)>0 .
\end{aligned}
$$

We obtain

$$
\begin{aligned}
0 & <\theta\left(\lambda_{2}, m\right)=\frac{1}{B\left(\lambda_{1}, \lambda_{2}\right)} \\
\cdot & \int_{0}^{(1+\eta)(1+\cos \beta) /(|m-\xi|+(m-\xi) \cos \alpha)} \frac{u^{\lambda_{2}-1}}{(1+u)^{\lambda}} d u \\
& <\frac{1}{B\left(\lambda_{1}, \lambda_{2}\right)} \int_{0}^{(1+\eta)(1+\cos \beta) /(|m-\xi|+(m-\xi) \cos \alpha)} u^{\lambda_{2}-1} d u \\
& =\frac{1}{\lambda_{2} B\left(\lambda_{1}, \lambda_{2}\right)}\left[\frac{(1+\eta)(1+\cos \beta)}{|m-\xi|+(m-\xi) \cos \alpha}\right]^{\lambda_{2}},
\end{aligned}
$$

and then we have (10) and (11).

In the same way, we still have the following.

Lemma 3. If $\lambda_{1} \leq 1$, then, for $k_{\alpha}\left(\lambda_{1}\right)=2 B\left(\lambda_{1}, \lambda_{2}\right) \csc ^{2} \alpha$, one has

$$
k_{\alpha}\left(\lambda_{1}\right)\left(1-\vartheta\left(\lambda_{1}, n\right)\right)<\omega\left(\lambda_{1}, n\right)<k_{\alpha}\left(\lambda_{1}\right), \quad, \quad|n| \in \mathbf{N},
$$

where

$$
\begin{aligned}
& \vartheta\left(\lambda_{1}, n\right):=\frac{1}{B\left(\lambda_{1}, \lambda_{2}\right)} \\
& \quad \cdot \int_{0}^{(1+\xi)(1+\cos \alpha) /(|n-\eta|+(n-\eta) \cos \beta)} \frac{u^{\lambda_{1}-1}}{(1+u)^{\lambda}} d u \\
& \quad=O\left(\frac{1}{[|n-\eta|+(n-\eta) \cos \beta]^{\lambda_{1}}}\right) \in(0,1),
\end{aligned}
$$

$|n| \in \mathbf{N}$.

Lemma 4. If $\zeta \in[0,1 / 2]$ and $\theta \in(0, \pi)$, then, for $\rho>0$,

$$
\begin{aligned}
& H_{\rho}(\zeta, \theta):=\sum_{|n|=1}^{\infty} \frac{1}{[|n-\zeta|+(n-\zeta) \cos \theta]^{1+\rho}} \\
& \quad=\frac{1+o(1)}{\rho}\left[\frac{1}{(1+\cos \theta)^{1+\rho}}+\frac{1}{(1-\cos \theta)^{1+\rho}}\right]
\end{aligned}
$$


Proof. We have

$$
\begin{aligned}
H_{\rho}(\zeta, \theta)= & \sum_{n=-1}^{-\infty} \frac{1}{[(n-\zeta)(\cos \theta-1)]^{1+\rho}} \\
& +\sum_{n=1}^{\infty} \frac{1}{[(n-\zeta)(\cos \theta+1)]^{1+\rho}} \\
= & \frac{1}{(1-\cos \theta)^{1+\rho}} \sum_{n=1}^{\infty} \frac{1}{(n+\zeta)^{1+\rho}} \\
& +\frac{1}{(1+\cos \theta)^{1+\rho}} \sum_{n=1}^{\infty} \frac{1}{(n-\zeta)^{1+\rho}}
\end{aligned}
$$

For $a=1 /(1-\zeta)^{1+\rho}$, we find

$$
\begin{aligned}
& H_{\rho}(\zeta, \theta) \leq\left[\frac{1}{(1-\cos \theta)^{1+\rho}}+\frac{1}{(1+\cos \theta)^{1+\rho}}\right][a \\
& \left.+\sum_{n=2}^{\infty} \frac{1}{(n-\zeta)^{1+\rho}}\right]<\left[\frac{1}{(1-\cos \theta)^{1+\rho}}\right. \\
& \left.+\frac{1}{(1+\cos \theta)^{1+\rho}}\right]\left[a+\int_{1}^{\infty} \frac{d y}{(y-\zeta)^{1+\rho}}\right] \\
& =\frac{1}{\rho}\left[\frac{1}{(1-\cos \theta)^{1+\rho}}+\frac{1}{(1+\cos \theta)^{1+\rho}}\right]\{1 \\
& \left.+\left[a \rho+\frac{1}{(1-\zeta)^{\rho}}-1\right]\right\} \\
& H_{\rho}(\zeta, \theta) \geq\left[\frac{1}{(1-\cos \theta)^{1+\rho}}+\frac{1}{(1+\cos \theta)^{1+\rho}}\right] \\
& \cdot \sum_{n=1}^{\infty} \frac{1}{(n+\zeta)^{1+\rho}}>\left[\frac{1}{(1-\cos \theta)^{1+\rho}}\right. \\
& \left.+\frac{1}{(1+\cos \theta)^{1+\rho}}\right] \int_{1}^{\infty} \frac{d y}{(y+\zeta)^{1+\rho}} \\
& =\frac{1+\left[(1+\zeta)^{-\rho}-1\right]}{\rho}\left[\frac{1}{(1-\cos \theta)^{1+\rho}}\right. \\
& \left.+\frac{1}{(1+\cos \theta)^{1+\rho}}\right] \text {. }
\end{aligned}
$$

Hence, we have (22).

\section{Main Results and Operation Expressions}

Theorem 5. If $\lambda_{1}, \lambda_{2} \leq 1, a_{m}, b_{n} \geq 0(|m|,|n| \in \mathbf{N})$,

$$
\begin{aligned}
0 & <\sum_{|m|=1}^{\infty}[|m-\xi|+(m-\xi) \cos \alpha]^{p\left(1-\lambda_{1}\right)-1} a_{m}^{p} \\
& <\infty
\end{aligned}
$$

$$
\begin{aligned}
0 & <\sum_{|n|=1}^{\infty}[|n-\eta|+(n-\eta) \cos \beta]^{q\left(1-\lambda_{2}\right)-1} b_{n}^{q} \\
& <\infty, \\
k\left(\lambda_{1}\right) & :=k_{\beta}^{1 / p}\left(\lambda_{1}\right) k_{\alpha}^{1 / q}\left(\lambda_{1}\right) \\
& =2 B\left(\lambda_{1}, \lambda_{2}\right) \csc ^{2 / p} \beta \csc ^{2 / q} \alpha,
\end{aligned}
$$

then one has the following equivalent inequalities:

$$
\begin{aligned}
& I: \sum_{|n|=1}^{\infty} \sum_{|m|=1}^{\infty} k(m, n) a_{m} b_{n}<k\left(\lambda_{1}\right) \\
& \cdot\left\{\sum_{|m|=1}^{\infty}[|m-\xi|+(m-\xi) \cos \alpha]^{p\left(1-\lambda_{1}\right)-1} a_{m}^{p}\right\}^{1 / p} \\
& \cdot\left\{\sum_{|n|=1}^{\infty}[|n-\eta|+(n-\eta) \cos \beta]^{q\left(1-\lambda_{2}\right)-1} b_{n}^{q}\right\}^{1 / q}, \\
& J:=\left\{\sum_{|n|=1}^{\infty}[|n-\eta|+(n-\eta) \cos \beta]^{p \lambda_{2}-1}\right. \\
& \\
&\left.\cdot\left\{\sum_{|m|=1}^{\infty} k(m, n) a_{m}\right)^{p}\right\}^{1 / p}<k\left(\lambda_{1}\right) \\
& \\
& \cdot\left\{\sum_{|m|=1}^{\infty}[|m-\xi|+(m-\xi) \cos \alpha]^{p\left(1-\lambda_{1}\right)-1} a_{m}^{p}\right\}^{1 / p} .
\end{aligned}
$$

Proof. By Hölder's inequality (cf. [23]) and (9), we have

$$
\begin{gathered}
\left(\sum_{|m|=1}^{\infty} k(m, n) a_{m}\right)^{p}=\left\{\sum_{|m|=1}^{\infty} k(m, n)\right. \\
\cdot \frac{[|m-\xi|+(m-\xi) \cos \alpha]^{\left(1-\lambda_{1}\right) / q}}{[|n-\eta|+(n-\eta) \cos \beta]^{\left(1-\lambda_{2}\right) / p}} a_{m} \\
\left.\cdot \frac{[|n-\eta|+(n-\eta) \cos \beta]^{\left(1-\lambda_{2}\right) / p}}{[|m-\xi|+(m-\xi) \cos \alpha]^{\left(1-\lambda_{1}\right) / q}}\right\} \\
\leq \sum_{|m|=1}^{\infty} k(m, n) \\
\cdot \frac{[|m-\xi|+(m-\xi) \cos \alpha]^{\left(1-\lambda_{1}\right) p / q}}{[|n-\eta|+(n-\eta) \cos \beta]^{1-\lambda_{2}}} a_{m}^{p}
\end{gathered}
$$

$\cdot\left\{\sum_{|m|=1}^{\infty} k(m, n) \frac{[|n-\eta|+(n-\eta) \cos \beta]^{\left(1-\lambda_{2}\right) q / p}}{[|m-\xi|+(m-\xi) \cos \alpha]^{1-\lambda_{1}}}\right\}^{p-1}$

$=\frac{\left(\omega\left(\lambda_{1}, n\right)\right)^{p-1}}{[|n-\eta|+(n-\eta) \cos \beta]^{p \lambda_{2}-1}} \sum_{|m|=1}^{\infty} k(m, n)$

$\cdot \frac{[|m-\xi|+(m-\xi) \cos \alpha]^{\left(1-\lambda_{1}\right) p / q}}{[|n-\eta|+(n-\eta) \cos \beta]^{1-\lambda_{2}}} a_{m}^{p}$. 
By (20), we have

$$
\begin{aligned}
J< & k_{\alpha}^{1 / q}\left(\lambda_{1}\right)\left\{\sum_{|n|=1}^{\infty} \sum_{|m|=1}^{\infty} k(m, n)\right. \\
& \left.\cdot \frac{[|m-\xi|+(m-\xi) \cos \alpha]^{\left(1-\lambda_{1}\right) p / q}}{[|n-\eta|+(n-\eta) \cos \beta]^{1-\lambda_{2}}} a_{m}^{p}\right\}^{1 / p} \\
& =k_{\alpha}^{1 / q}\left(\lambda_{1}\right)\left\{\sum_{|m|=1}^{\infty} \sum_{|n|=1}^{\infty} k(m, n)\right. \\
& \left.\cdot \frac{[|m-\xi|+(m-\xi) \cos \alpha]^{\left(1-\lambda_{1}\right) p / q}}{[|n-\eta|+(n-\eta) \cos \beta]^{1-\lambda_{2}}} a_{m}^{p}\right\}^{1 / p} \\
= & k_{\alpha}^{1 / q}\left(\lambda_{1}\right)\left\{\sum_{|m|=1}^{\infty} \omega\left(\lambda_{2}, m\right)[|m-\xi|+(m-\xi)\right. \\
\cdot & \left.\cos \alpha]^{p\left(1-\lambda_{1}\right)-1} a_{m}^{p}\right\}^{1 / p} \cdot
\end{aligned}
$$

By (10), we have (27).

By Hölder's inequality (cf. [23]), we have

$$
\begin{aligned}
I= & \sum_{|n|=1}^{\infty}\left\{[|n-\eta|+(n-\eta) \cos \beta]^{\lambda_{2}-1 / p}\right. \\
& \left.\cdot \sum_{|m|=1}^{\infty} k(m, n) a_{m}\right\}[|n-\eta|+(n-\eta) \cos \beta]^{1 / p-\lambda_{2}} b_{n} \\
& \leq J\left\{\sum_{|n|=1}^{\infty}[|n-\eta|+(n-\eta) \cos \beta]^{q\left(1-\lambda_{2}\right)-1} b_{n}^{q}\right\}^{1 / q} .
\end{aligned}
$$

Then by (27), we have (26).

On the other hand, assuming that (26) is valid, we set

$$
\begin{aligned}
b_{n}:= & {[|n-\eta|+(n-\eta) \cos \beta]^{p \lambda_{2}-1} } \\
& \cdot\left(\sum_{|m|=1}^{\infty} k(m, n) a_{m}\right)^{p-1}, \quad|n| \in \mathbf{N} .
\end{aligned}
$$

Then it follows that

$$
J=\left\{\sum_{|n|=1}^{\infty}[|n-\eta|+(n-\eta) \cos \beta]^{q\left(1-\lambda_{2}\right)-1} b_{n}^{q}\right\}^{1 / p} .
$$

By (29), we find $J<\infty$. If $J=0$, then (27) is evidently valid; if $J>0$, then, by (26), we have

$$
\begin{aligned}
0< & \sum_{|n|=1}^{\infty}[|n-\eta|+(n-\eta) \cos \beta]^{q\left(1-\lambda_{2}\right)-1} b_{n}^{q}=J^{p}=I \\
& <k\left(\lambda_{1}\right)
\end{aligned}
$$

$$
\begin{aligned}
& \cdot\left\{\sum_{|m|=1}^{\infty}[|m-\xi|+(m-\xi) \cos \alpha]^{p\left(1-\lambda_{1}\right)-1} a_{m}^{p}\right\}^{1 / p} \\
& \cdot\left\{\sum_{|n|=1}^{\infty}[|n-\eta|+(n-\eta) \cos \beta]^{q\left(1-\lambda_{2}\right)-1} b_{n}^{q}\right\}^{1 / q}, \\
J & \left\{\sum_{|n|=1}^{\infty}[|n-\eta|+(n-\eta) \cos \beta]^{q\left(1-\lambda_{2}\right)-1} b_{n}^{q}\right\}^{1 / p} \\
& <k\left(\lambda_{1}\right) \\
& \cdot\left\{\sum_{|m|=1}^{\infty}[|m-\xi|+(m-\xi) \cos \alpha]^{p\left(1-\lambda_{1}\right)-1} a_{m}^{p}\right\}^{1 / p} ;
\end{aligned}
$$

namely, (27) follows, which is equivalent to (26).

Theorem 6. As regards the assumptions of Theorem 5, the constant factor $k\left(\lambda_{1}\right)$ in (26) and (27) is the best possible one.

Proof. For any $\varepsilon \in\left(0, q \lambda_{2}\right)$, we set $\tilde{\lambda}_{1}=\lambda_{1}+\varepsilon / q, \tilde{\lambda}_{2}=\lambda_{2}-$ $\varepsilon / q(\in(0,1))$, and

$$
\begin{aligned}
\tilde{a}_{m} & :=[|m-\xi|+(m-\xi) \cos \alpha]^{\left(\lambda_{1}-\varepsilon / p\right)-1} \\
& =[|m-\xi|+(m-\xi) \cos \alpha]^{\widetilde{\lambda}_{1}-\varepsilon-1} \quad(|m| \in \mathbf{N}), \\
\tilde{b}_{n} & :=[|n-\eta|+(n-\eta) \cos \beta]^{\left(\lambda_{2}-\varepsilon / q\right)-1} \\
& =[|n-\eta|+(n-\eta) \cos \beta]^{\widetilde{\lambda}_{2}-1} \quad(|n| \in \mathbf{N}) .
\end{aligned}
$$

Then by (22) and (10), we find

$$
\begin{aligned}
\tilde{I}_{1} & =\left\{\sum_{|m|=1}^{\infty}[|m-\xi|+(m-\xi) \cos \alpha]^{p\left(1-\lambda_{1}\right)-1} \tilde{a}_{m}^{p}\right\}^{1 / p} \\
& \cdot\left\{\sum_{|n|=1}^{\infty}[|n-\eta|+(n-\eta) \cos \beta]^{q\left(1-\lambda_{2}\right)-1} \widetilde{b}_{n}^{q}\right\}^{1 / q} \\
& =\left\{\sum_{|m|=1}^{\infty} \frac{1}{[|m-\xi|+(m-\xi) \cos \alpha]^{1+\varepsilon}}\right\}^{1 / p} \\
& \cdot\left\{\sum_{|n|=1}^{\infty} \frac{1}{[|n-\eta|+(n-\eta) \cos \beta]^{1+\varepsilon}}\right\}^{1 / q} \\
& =\frac{1}{\varepsilon}\left[\frac{1}{(1+\cos \alpha)^{1+\varepsilon}}+\frac{1}{(1-\cos \alpha)^{1+\varepsilon}}\right]^{1 / p} \\
& \cdot\left[\frac{1}{(1+\cos \beta)^{1+\varepsilon}}+\frac{1}{(1-\cos \beta)^{1+\varepsilon}}\right]^{1 / q}\left(1+o_{1}(1)\right)^{1 / p} \\
& \cdot\left(1+o_{2}(1)\right)^{1 / q},
\end{aligned}
$$




$$
\begin{aligned}
& \widetilde{I}:=\sum_{|n|=1}^{\infty} \sum_{|m|=1}^{\infty} k(m, n) \widetilde{a}_{m} \tilde{b}_{n}=\sum_{|m|=1}^{\infty} \sum_{|m|=1}^{\infty} k(m, n) \\
& \cdot \frac{[|m-\xi|+(m-\xi) \cos \alpha]^{\widetilde{\lambda}_{1}-\varepsilon-1}}{[|n-\eta|+(n-\eta) \cos \beta]^{1-\tilde{\lambda}_{2}}} \\
& =\sum_{|m|=1}^{\infty} \frac{\omega\left(\tilde{\lambda}_{2}, m\right)}{[|m-\xi|+(m-\xi) \cos \alpha]^{\varepsilon+1}} \geq k_{\beta}\left(\tilde{\lambda}_{1}\right) \\
& \cdot \sum_{|m|=1}^{\infty} \frac{1-\theta\left(\tilde{\lambda}_{2}, m\right)}{[|m-\xi|+(m-\xi) \cos \alpha]^{\varepsilon+1}}=k_{\beta}\left(\tilde{\lambda}_{1}\right) \\
& \left\{\sum_{|m|=1}^{\infty} \frac{1}{[|m-\xi|+(m-\xi) \cos \alpha]^{\varepsilon+1}}\right. \\
& \left.-\sum_{|m|=1}^{\infty} \frac{1}{O\left([|m-\xi|+(m-\xi) \cos \alpha]^{\left(\varepsilon / p+\lambda_{2}\right)+1}\right)}\right\} \\
& =\frac{k_{\beta}\left(\tilde{\lambda}_{1}\right)}{\varepsilon}\left\{\left[\frac{1}{(1+\cos \alpha)^{1+\varepsilon}}+\frac{1}{(1-\cos \alpha)^{1+\varepsilon}}\right](1\right. \\
& \left.\left.+o_{1}(1)\right)-\varepsilon O(1)\right\} \text {. }
\end{aligned}
$$

If there exists a constant $k \leq k\left(\lambda_{1}\right)$, such that (26) is valid when replacing $k\left(\lambda_{1}\right)$ by $k$, then, in particular, we have $\varepsilon \widetilde{I}<$ $k \widetilde{I}_{1}$; namely,

$$
\begin{aligned}
& k_{\beta}\left(\tilde{\lambda}_{1}\right) \\
& \cdot\left\{\left[\frac{1}{(1+\cos \alpha)^{1+\varepsilon}}+\frac{1}{(1-\cos \alpha)^{1+\varepsilon}}\right]\left(1+o_{1}(1)\right)\right. \\
& -\varepsilon O(1)\}<k\left[\frac{1}{(1+\cos \alpha)^{1+\varepsilon}}\right. \\
& \left.+\frac{1}{(1-\cos \alpha)^{1+\varepsilon}}\right]^{1 / p}\left(1+o_{1}(1)\right)^{1 / p} \\
& \cdot\left[\frac{1}{(1+\cos \beta)^{1+\varepsilon}}+\frac{1}{(1-\cos \beta)^{1+\varepsilon}}\right]^{1 / q}(1 \\
& \left.+o_{2}(1)\right)^{1 / q} .
\end{aligned}
$$

It follows that

$$
\begin{aligned}
& 4 B\left(\lambda_{1}, \lambda_{2}\right) \csc ^{2} \beta \csc ^{2} \alpha \leq 2 k \csc ^{2 / p} \alpha \csc ^{2 / q} \beta \\
&\left(\varepsilon \longrightarrow 0^{+}\right) ;
\end{aligned}
$$

namely, $k\left(\lambda_{1}\right)=2 B\left(\lambda_{1}, \lambda_{2}\right) \csc ^{2 / p} \beta \csc ^{2 / q} \alpha \leq k$. Hence, $k=$ $k\left(\lambda_{1}\right)$ is the best possible constant factor of (26). The constant factor $k\left(\lambda_{1}\right)$ in (27) is still the best possible one. Otherwise, we would reach a contradiction by (30) that the constant factor in (26) is not the best possible one.
We set functions $\Phi(m)$ and $\Psi(n)$ as follows:

$$
\begin{aligned}
& \Phi(m):=[|m-\xi|+(m-\xi) \cos \alpha]^{p\left(1-\lambda_{1}\right)-1} \\
& \quad(|m| \in \mathbf{N}), \\
& \Psi(n):=[|n-\eta|+(n-\eta) \cos \beta]^{q\left(1-\lambda_{2}\right)-1}
\end{aligned}
$$

$(|n| \in \mathbf{N})$,

wherefrom $\Psi^{1-p}(n)=[|n-\eta|+(n-\eta) \cos \beta]^{p \lambda_{2}-1}(|n| \in \mathbf{N})$. We also set the following weight normed spaces:

$$
\begin{aligned}
l_{p, \Phi} & :=\left\{a=\left\{a_{m}\right\}_{|m|=1}^{\infty} ;\|a\|_{p, \Phi}\right. \\
& \left.=\left(\sum_{|m|=1}^{\infty} \Phi(m)\left|a_{m}\right|^{p}\right)^{1 / p}<\infty\right\}, \\
l_{q, \Psi} & :=\left\{b=\left\{b_{n}\right\}_{|n|=1}^{\infty} ;\|b\|_{q, \Psi}=\left(\sum_{|n|=1}^{\infty} \Psi(n)\left|b_{n}\right|^{q}\right)^{1 / q}\right. \\
& <\infty\},
\end{aligned}
$$

$$
\begin{aligned}
l_{p, \Psi^{1-p}} & :=\left\{c=\left\{c_{n}\right\}_{|n|=1}^{\infty} ;\|c\|_{p, \Psi^{1-p}}\right. \\
& \left.=\left(\sum_{|n|=1}^{\infty} \Psi^{1-p}(n)\left|c_{n}\right|^{p}\right)^{1 / p}<\infty\right\} .
\end{aligned}
$$

Then, for $a=\left\{a_{m}\right\}_{|m|=1}^{\infty} \in l_{p, \Phi}, c=\left\{c_{n}\right\}_{|n|=1}^{\infty}$, and $c_{n}=\sum_{|m|=1}^{\infty} k(m, n) a_{m}$, in view of (27), we have $\|c\|_{p, \Psi^{1-p}}<$ $k\left(\lambda_{1}\right)\|a\|_{p, \Phi} ;$ namely, $c \in l_{p, \Psi^{1-p}}$

Definition 7. Define a Hilbert-type operator $T: l_{p, \Phi} \rightarrow l_{p, \Psi^{1-p}}$ as follows: for any $a=\left\{a_{m}\right\}_{|m|=1}^{\infty} \in l_{p, \Phi}$, there exists a unique representation $c=T a \in l_{p, \Psi^{1-p}}$. One also defines the formal inner product of $T a$ and $b=\left\{b_{n}\right\}_{|n|=1}^{\infty} \in l_{q, \Psi}\left(b_{n} \geq 0\right)$ as follows:

$$
(T a, b):=\sum_{|n|=1}^{\infty} \sum_{|m|=1}^{\infty} k(m, n) a_{m} b_{n} .
$$

Then for $a_{m} \geq 0(|m| \in \mathbf{N})$, we may rewrite (26) and (27) as follows:

$$
(T a, b)<k\left(\lambda_{1}\right)\|a\|_{p, \Phi}\|b\|_{q, \Psi},
$$$$
\|T a\|_{p, \Psi^{1-p}}<k\left(\lambda_{1}\right)\|a\|_{p, \Phi} .
$$

We define the norm of operator $T$ as follows:

$$
\|T\|:=\sup _{a(\neq \theta) \in l_{p, \Phi}} \frac{\|T a\|_{p, \Psi^{1-p}}}{\|a\|_{p, \Phi}} .
$$


Since, by Theorem 6 , the constant factor $k\left(\lambda_{1}\right)$ in (42) is the best possible one, we have

$$
\|T\|=k\left(\lambda_{1}\right)=2 B\left(\lambda_{1}, \lambda_{2}\right) \csc ^{2 / p} \beta \csc ^{2 / q} \alpha .
$$

Remark 8. (i) For $\xi=\eta=0$, (26) reduces to

$$
\begin{aligned}
& \sum_{|n|=1}^{\infty} \sum_{|m|=1}^{\infty} \frac{1}{(|m|+m \cos \alpha+|n|+n \cos \beta)^{\lambda}} a_{m} b_{n} \\
& <k\left(\lambda_{1}\right)\left[\sum_{|m|=1}^{\infty}(|m|+m \cos \alpha)^{p\left(1-\lambda_{1}\right)-1} a_{m}^{p}\right]^{1 / p} \\
& .\left[\sum_{|n|=1}^{\infty}(|n|+n \cos \beta)^{q\left(1-\lambda_{2}\right)-1} b_{n}^{q}\right]^{1 / q} .
\end{aligned}
$$

Hence, (26) is a more accurate inequality of (45). In particular, for $\alpha=\beta=\pi / 2$ in (45), we have the following new inequality:

$$
\begin{gathered}
\sum_{|n|=1}^{\infty} \sum_{|m|=1}^{\infty} \frac{a_{m} b_{n}}{(|m|+|n|)^{\lambda}}<2 B\left(\lambda_{1}, \lambda_{2}\right) \\
\cdot\left[\sum_{|m|=1}^{\infty}|m|^{p\left(1-\lambda_{1}\right)-1} a_{m}^{p}\right]^{1 / p} \\
\cdot\left[\sum_{|n|=1}^{\infty}|n|^{q\left(1-\lambda_{2}\right)-1} b_{n}^{q}\right]^{1 / q} .
\end{gathered}
$$

(ii) For $\alpha=\beta=\pi / 2$ in (26), we have (5). For $a_{-m}=a_{m}$ and $b_{-n}=b_{n}(m, n \in \mathbf{N})$, (5) reduces to

$$
\begin{aligned}
\sum_{n=1}^{\infty} & \sum_{m=1}^{\infty}\left[\frac{1}{(m+n-\xi-\eta)^{\lambda}}+\frac{1}{(m+n+\xi-\eta)^{\lambda}}\right. \\
& \left.+\frac{1}{(m+n+\xi+\eta)^{\lambda}}+\frac{1}{(m+n-\xi+\eta)^{\lambda}}\right] a_{m} b_{n} \\
& <2 B\left(\lambda_{1}, \lambda_{2}\right) \\
& \cdot\left\{\sum_{m=1}^{\infty}\left[(m-\xi)^{p\left(1-\lambda_{1}\right)-1}+(m+\xi)^{p\left(1-\lambda_{1}\right)-1}\right] a_{m}^{p}\right\}^{1 / p} \\
& \cdot\left\{\sum_{n=1}^{\infty}\left[(n-\eta)^{q\left(1-\lambda_{2}\right)-1}+(n+\eta)^{q\left(1-\lambda_{2}\right)-1}\right] b_{n}^{q}\right\}^{1 / q} .
\end{aligned}
$$

In particular, for $\xi=\eta \in[0,1 / 2]$, we have

$$
\begin{aligned}
& \sum_{n=1}^{\infty} \sum_{m=1}^{\infty}\left[\frac{1}{(m+n-2 \eta)^{\lambda}}+\frac{2}{(m+n)^{\lambda}}\right. \\
& \left.+\frac{1}{(m+n+2 \eta)^{\lambda}}\right] a_{m} b_{n}<2 B\left(\lambda_{1}, \lambda_{2}\right) \\
& \cdot\left\{\sum_{m=1}^{\infty}\left[(m-\eta)^{p\left(1-\lambda_{1}\right)-1}+(m+\eta)^{p\left(1-\lambda_{1}\right)-1}\right]\right. \\
& \left.\cdot a_{m}^{p}\right\}^{1 / p}\left\{\sum_{n=1}^{\infty}\left[(n-\eta)^{q\left(1-\lambda_{2}\right)-1}+(n+\eta)^{q\left(1-\lambda_{2}\right)-1}\right]\right. \\
& \left.\cdot b_{n}^{q}\right\}^{1 / q} \cdot \\
& \text { If } \eta=0, \text { then }(48) \text { reduces to } \\
& \sum_{n=1}^{\infty} \sum_{m=1}^{\infty} \frac{a_{m} b_{n}}{(m+n)^{\lambda}}<B\left(\lambda_{1}, \lambda_{2}\right)\left[\sum_{m=1}^{\infty} m^{p\left(1-\lambda_{1}\right)-1} a_{m}^{p}\right]^{1 / p} \\
& \cdot\left[\sum_{n=1}^{\infty} n^{q\left(1-\lambda_{2}\right)-1} b_{n}^{q}\right]^{1 / q},
\end{aligned}
$$

which is an extension of (1).

\section{Competing Interests}

The authors declare that they have no competing interests.

\section{Acknowledgments}

This work is supported by Science and Technology Planning Project of Guangdong Province (no. 2013A011403002) and Appropriative Researching Fund for Professors and Doctors, Guangdong University of Education (no. 2015ARF25). The authors are grateful for their help.

\section{References}

[1] G. H. Hardy, "Note on a theorem of Hilbert concerning series of positive terms," Proceedings of the London Mathematical Society, vol. 23, no. 2, pp. 45-46, 1925, Records of Procceedings.

[2] G. H. Hardy, J. E. Littlewood, and G. P, Cambridge University Press, 1934

[3] B. Yang, Discrete Hilbert-Type Inequalities, Bentham Science, Dubai, United Arab Emirates, 2011.

[4] Z. Wang and D. Guo, Introduction to Special Functions, Science Press, Beijing, China, 1979.

[5] D. S. Mitrinović, J. E. Pečarić, and A. M. Fink, Inequalities Involving Functions and Their Integrals and Derivatives, Kluwer Academic, Boston, Mass, USA, 1991.

[6] M. Gao and B. Yang, "On the extended Hilbert's inequality," Proceedings of the American Mathematical Society, vol. 126, no. 3, pp. 751-759, 1998. 
[7] Y. Hong, "All-sided generalization about Hardy-Hilbert integral inequalities," Acta Mathematica Sinica, vol. 44, no. 4, pp. 619626, 2001.

[8] K. Zhang, "A bilinear inequality," Journal of Mathematical Analysis and Applications, vol. 271, no. 1, pp. 288-296, 2002.

[9] A. Benyi and C. Oh, "Best constants for certain multilinear integral operators," Journal of Inequalities and Applications, vol. 2006, Article ID 28582, 12 pages, 2006.

[10] J. Kuang and L. Debnath, "On Hilbert's type integral inequalities on the weighted Orlicz spaces," Pacific Journal of Applied Mathematics, vol. 1, no. 1, pp. 95-104, 2007.

[11] Y. Li and B. He, "On inequalities of Hilbert's type," Bulletin of the Australian Mathematical Society, vol. 76, no. 1, pp. 1-13, 2007.

[12] L. E. Azar, "On some extensions of Hardy-Hilbert's inequality and applications," Journal of Inequalities and Applications, vol. 2008, Article ID 546829, 2008.

[13] W. Zhong, "The Hilbert-type integral inequalities with a homogeneous kernel of - $\lambda$-degree," Journal of Inequalities and Applications, vol. 2008, Article ID 917392, 12 pages, 2008.

[14] J. Jin and L. Debnath, "On a Hilbert-type linear series operator and its applications," Journal of Mathematical Analysis and Applications, vol. 371, no. 2, pp. 691-704, 2010.

[15] Q. Huang, "On a multiple Hilbert's inequality with parameters," Journal of Inequalities and Applications, vol. 2010, Article ID 309319, 12 pages, 2010.

[16] B. Yang and M. Krnić, "On the norm of a multi-dimensional Hilberttype operator," Sarajevo Journal of Mathematics, vol. 7, no. 20, pp. 223-243, 2011.

[17] M. Krnić and P. Vuković, "On a multidimensional version of the Hilbert type inequality," Analysis Mathematica, vol. 38, no. 4, pp. 291-303, 2012.

[18] V. Adiyasuren, T. Batbold, and M. Krnić, "Half-discrete Hilberttype inequalities with mean operators, the best constants, and applications," Applied Mathematics and Computation, vol. 231, pp. 148-159, 2014.

[19] Q. Huang, "A new extension of a Hardy-Hilbert-type inequality," Journal of Inequalities and Applications, vol. 2015, article 397, 12 pages, 2015.

[20] B. He and Q. Wang, "A multiple Hilbert-type discrete inequality with a new kernel and best possible constant factor," Journal of Mathematical Analysis and Applications, vol. 431, no. 2, pp. 889902, 2015.

[21] Y. Shi and B. Yang, "A new Hardy-Hilbert-type inequality with multiparameters and a best possible constant factor," Journal of Inequalities and Applications, vol. 2015, article 380, 2015.

[22] V. Adiyasuren, Ts. Batbold, and M. Krnić, "Multiple Hilberttype inequalities involving some differential operators," Banach Journal of Mathematical Analysis, vol. 10, no. 2, pp. 320-337, 2016.

[23] J. Kuang, Applied Inequalities, Shangdong Science and Technology Press, Jinan, China, 2004. 


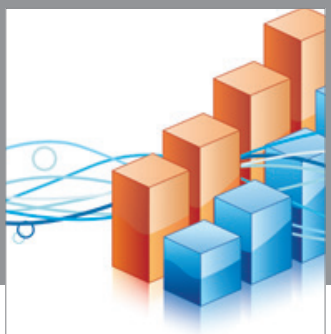

Advances in

Operations Research

vatem alat4

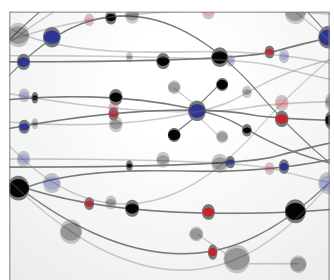

\section{The Scientific} World Journal
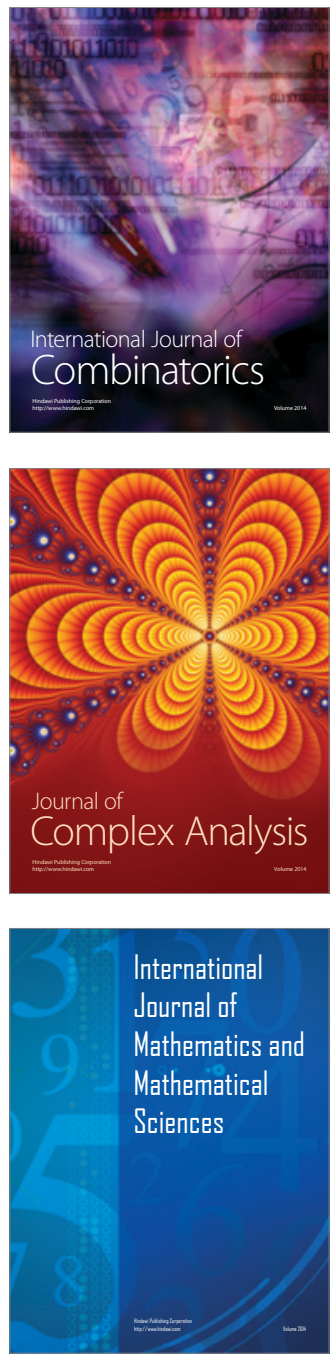
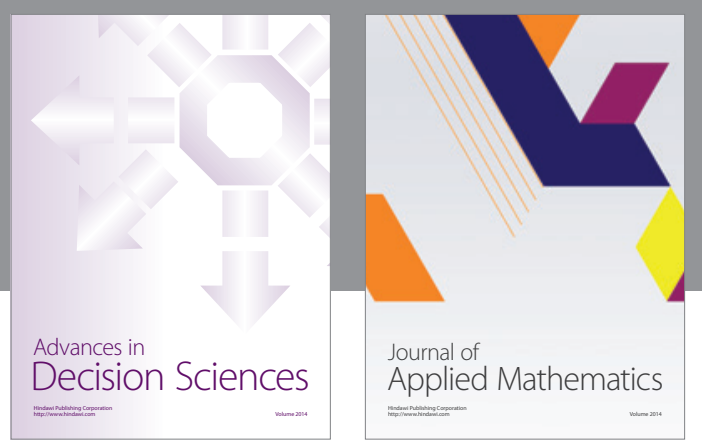

Algebra

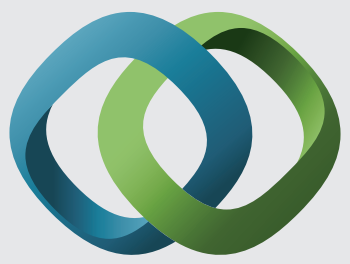

\section{Hindawi}

Submit your manuscripts at

http://www.hindawi.com
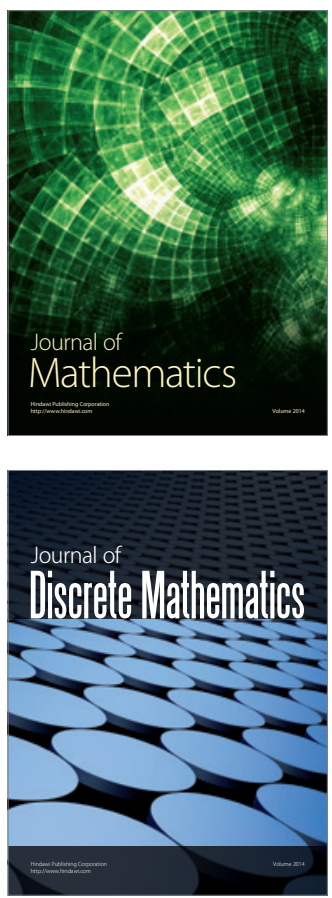

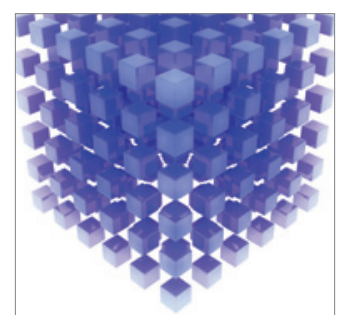

Mathematical Problems in Engineering
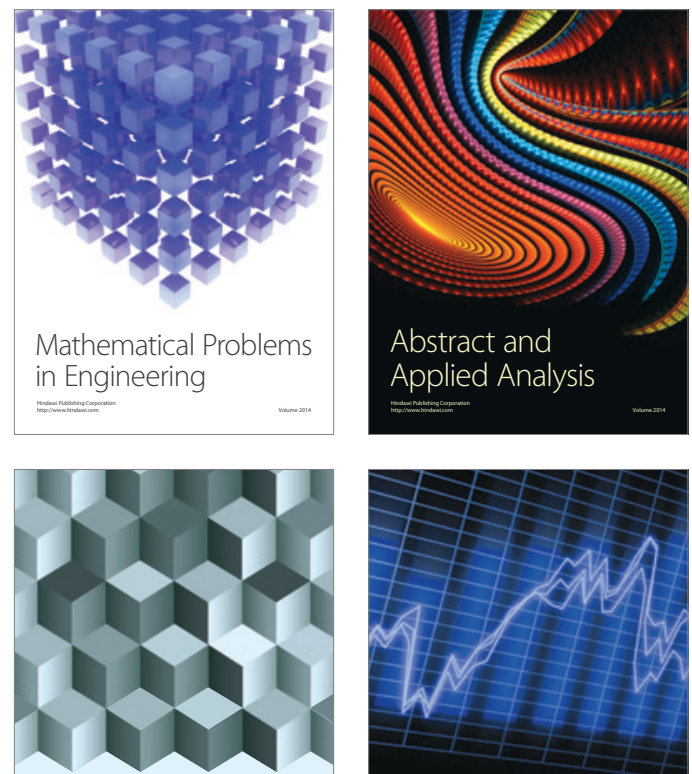

Journal of

Function Spaces

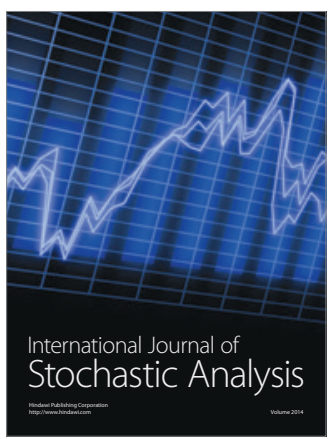

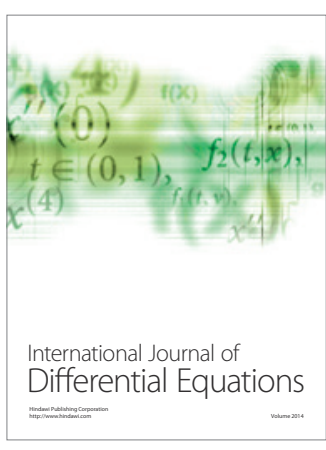
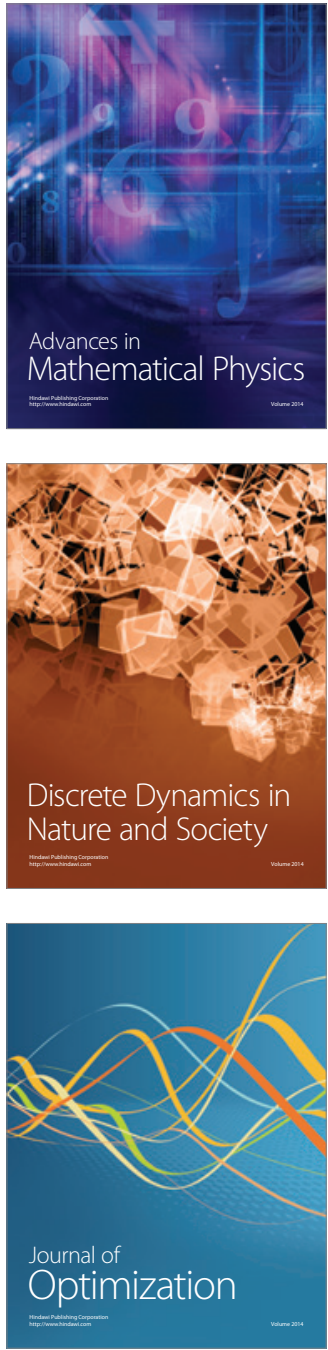